\title{
Predição da energia de ativação de fluxo viscoso dos líquidos iônicos imidazólicos $[\mathrm{HMIM}]\left[\mathrm{PF}_{6}\right]$ e $[\mathrm{BMIM}]\left[\mathrm{Tf}_{2} \mathrm{~N}\right]$
}

\author{
César Augusto CANCIAM* \\ * Mestre em Engenharia Química, professor lotado no Departamento Acadêmico de Engenharia Química da \\ Universidade Tecnológica Federal do Paraná - Câmpus Ponta Grossa, canciam@utfpr.edu.br
}

Recebido em: 27/12/2013 - Aprovado em: 14/09/2014 - Disponibilizado em: 15/12/2014

RESUMO: Os líquidos iônicos têm despertado grande interesse principalmente com relação à potencialidade de substituir os solventes orgânicos convencionais nos mais diversos processos. O presente trabalho teve por objetivo predizer a energia de ativação de fluxo viscoso dos líquidos iônicos imidazólicos hexafluorofosfato de 1-metil-3hexilimidazólio ([HMIM] $\left[\mathrm{PF}_{6}\right]$ ) e bis(trifluorosulfonilo)imideto de 1-butil-3-metilimidazólio ([BMIM][Tf $\left.{ }_{2} \mathrm{~N}\right]$ ). Para tanto, realizou-se uma análise de regressão linear de dados experimentais da viscosidade de cada líquido iônico imidazólico em função da temperatura a 0,1MPa. Esses dados experimentais foram encontrados na literatura. Os valores da energia de ativação de fluxo viscoso foram iguais a 46,6211 kJ.mol ${ }^{-1}$ para o [HMIM] $\left.\mathrm{PF}_{6}\right]$ e $29,1744 \mathrm{~kJ} . \mathrm{mol}^{-1} \mathrm{para} \mathrm{o}^{\circ}$ [BMIM] [Tf $\left.f_{2} \mathrm{~N}\right]$. Nos dois casos, os coeficientes de correlação foram próximos da unidade.

PALAVRAS-CHAVE: Viscosidade. Temperatura. Efeito. Líquidos Iônicos. Regressão linear.

\section{Prediction of activation energy for viscous flow of imidazolium ionic liquids

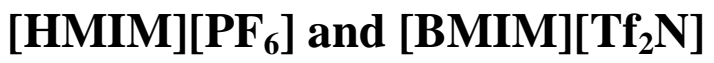

ABSTRACT: The ionic liquids have attracted great interest especially in relation to potencial to replace conventional organic solvents in various processes. This work aimed to predict values for the activation energy for viscous flow of imidazolium ionic liquids 1-methyl-3-hexylimidazolium hexafluorophosphate ([HMIM][PF $]$ ) and 1-butyl-3methylimidazolium bis(trifluorosulfonyl)imide ([BMIM] $\left.\left[\mathrm{Tf}_{2} \mathrm{~N}\right]\right)$. For this purpose, there was a linear regression analysis of experimental data of the viscosity of each imidazolium ionic liquid according to the temperature at $0.1 \mathrm{MPa}$. These experimental data were found in the literature. The values of activation energy for viscous flow were equal to $46.6211 \mathrm{~kJ} . \mathrm{mol}-1$ for $[\mathrm{HMIM}]\left[\mathrm{PF}_{6}\right]$ and $29.1744 \mathrm{~kJ} \cdot \mathrm{mol}^{-1}$ for $[\mathrm{BMIM}]\left[\mathrm{Tf} \mathrm{f}_{2} \mathrm{~N}\right]$. In both cases, the correlation coefficients were close to unity.

KEYWORDS: Viscosity. Temperature. Effect. Ionic liquids. Linear regression.

\section{INTRODUÇÃO}

Os líquidos iônicos pertencem à classe dos sais orgânicos e são constituídos por cátions orgânicos relativamente grandes e ânions orgânicos ou inorgânicos menores. Essa assimetria na sua composição química reduz a energia reticular do sal, impedindo a formação de uma estrutura cristalina ordenada (VALENTE, 2008).

De forma geral, os líquidos iônicos apresentam uma energia livre de Gibbs de solvatação negativa, resultando em uma baixa energia reticular e uma grande entropia, implicando em baixo ponto de fusão. Ou seja, o estado líquido é termodinamicamente 
favorável para esses compostos, mesmo em baixas temperaturas (RODRIGUES, 2010).

A ausência de uma estrutura cristalina ordenada confere aos líquidos iônicos: elevada capacidade calorífica, volatilidade extremamente baixa, inflamabilidade e toxicidade baixas; estabilidade térmica e condutividade iônica elevadas, larga gama de temperatura no estado líquido, densidades superiores à da água, viscosidades comparadas a óleos, miscibilidade em uma larga gama de concentrações em substâncias polares e apolares e capacidade de absorver moléculas menores (VALENTE, 2008; ANDO e SANTOS, 2011).

Devido à reduzida volatilidade e baixa toxicidade, os líquidos iônicos são ecologicamente aceitáveis e considerados “amigos" do ambiente (SILVA et al., 2005; VALENTE, 2008). Lima (2011) classifica os líquidos iônicos como "meios alternativos ambientalmente seguros", pois oferecem poucos riscos ao ambiente durante o processo, além de possibilitar a separação dos produtos e a recuperação do solvente com baixo consumo de energia.

Consorti e coautores (2001) comentam sobre a utilização dos líquidos iônicos como solventes (em reações orgânicas, em catálise bifásica, para a extração líquido-líquido e para a análise espectroscópica de compostos metálicos) e como fase estacionária para cromatografia gasosa.

Utilizam-se líquidos iônicos como tensoativos, catalisadores ácidos nas reações orgânicas e cossolventes em reações enzimáticas. Em eletroquímica, os líquidos iônicos são eletrólitos para diversas tecnologias (em baterias secundárias, células fotoelétricas e de combustíveis), capacitores elétricos de dupla camada e outros dispositivos eletroquímicos (CONSORTI et al, 2001; VALENTE, 2008).

Os líquidos iônicos, em comparação com os líquidos moleculares convencionais, possuem estrutura mais organizada, apresentando regiões com características bastante distintas. São compostos com regiões polares e apolares, onde ocorrem interações intermoleculares dos tipos eletrostáticas, de hidrogênio e de van der Waals. Como exemplo, enquanto os líquidos moleculares convencionais possuem um arranjo estrutural somente a curta distância (primeiros vizinhos) e por um curto espaço de tempo; nos sólidos cristalinos a organização estrutural pode ser vista a longas distâncias e por grandes períodos de tempo. Nesse sentido, os líquidos iônicos representam um meio termo, pois apresentam arranjo estrutural a médias distâncias e por tempos relativamente longos para um líquido (RODRIGUES, 2010). 
Os líquidos iônicos imidazólicos apresentam-se como estruturas supramoleculares poliméricas, altamente organizadas, em que cada cátion imidazólio é circundado de ânions e cada ânion é circundado de cátions imidazólios, através das ligações de hidrogênio existentes entre os hidrogênios do ciclo imidazólico e os ânions (MIGLIORINI, 2009).

Com relação aos líquidos iônicos imidazólicos, existe um grande interesse na sua utilização, pois apresentam: estabilidade ao ar e água, baixa inflamabilidade, alta estabilidade térmica, pressão de vapor muito baixa, amplas janelas eletroquímicas, altas condutividades elétrica e térmica, altas mobilidades iônicas e fácil recuperação e reutilização (DUPONT, 2004; MIGLIORINI, 2009).

Os líquidos iônicos imidazólicos $[\mathrm{HMIM}]\left[\mathrm{PF}_{6}\right] \quad$ (fórmula molecular $\mathrm{C}_{10} \mathrm{H}_{19} \mathrm{~N}_{2} \mathrm{PF}_{6}$ ) e [BMIM] $\left[\mathrm{Tf}_{2} \mathrm{~N}\right]$ (fórmula molecular $\mathrm{C}_{10} \mathrm{H}_{15} \mathrm{~N}_{3} \mathrm{O}_{4} \mathrm{~S}_{2} \mathrm{~F}_{6}$ ) apresentam-se como líquidos viscosos, de coloração que vai de incolor a levemente amarelada. São insolúveis em água e sem odor característico. $\mathrm{O}$ ponto de fusão do $[\mathrm{HMIM}]\left[\mathrm{PF}_{6}\right]$ é $-73,5^{\circ} \mathrm{C}$ e do [BMIM] $\left[\mathrm{Tf}_{2} \mathrm{~N}\right]$ é $-5^{\circ} \mathrm{C}$ (ROGERS et al, 2002).

A determinação da viscosidade de líquidos tem como base na Teoria do Estado Ativado de Eyring. Essa teoria considera que um líquido é constituído por moléculas intercaladas por posições desocupadas (vacâncias), e essas moléculas, mesmo com o líquido em repouso, movem-se com o propósito de ocuparem as vacâncias adjacentes a elas. A viscosidade está relacionada com a força que tende a se opor a esse movimento, sendo uma medida de fricção interna do fluido. Dessa forma, quanto maior a barreira potencial que uma molécula terá que vencer a fim de "saltar" para a vacância adjacente, maior é a viscosidade. Essa barreira potencial é conhecida como energia de ativação de fluxo viscoso (RIBEIRO et al, 2005; SILVA et al, 2007).

A temperatura é um parâmetro relacionado com a energia interna de uma substância. Vários estudos têm demonstrado que a viscosidade de um líquido é altamente influenciada por mudanças na temperatura (OLIVEIRA et al, 2009).

Shames (1999) comenta que a viscosidade é diretamente proporcional à força de atração entre as moléculas. Com o aumento da temperatura, essa força de atração diminui, diminuindo também a viscosidade. Dessa maneira, observa-se nos líquidos que a viscosidade diminui com o aumento da temperatura.

A redução da viscosidade dos líquidos com o aumento da temperatura é atribuída ao aumento das distâncias intermoleculares 
provocadas durante $\mathrm{o}$ aquecimento. $\mathrm{O}$ aumento das distâncias intermoleculares reduz as forças atrativas entre as moléculas, diminuindo a viscosidade (GRANJEIRO et al., 2007).

Giap (2010) comenta que o efeito da temperatura sobre a viscosidade é modelado por uma relação do tipo Arrhenius, conforme a Equação 1.

$$
\eta=\eta_{\infty} \cdot e^{\left(\frac{E_{a}}{R \cdot T}\right)}
$$

Em que $E_{a}$ corresponde à energia de ativação de fluxo viscoso; $R$, à constante universal dos gases ideais; $T$, à temperatura absoluta; $\quad \eta$, à viscosidade e $\eta_{\infty}$, à viscosidade quando a temperatura tende ao infinito.

Gratão e coautores (2004) comentam que a Equação 1 pode ser reescrita na forma de:

$$
\ln \eta=\ln \eta_{\infty}+\left(\frac{E_{a}}{R}\right) \cdot \frac{1}{T}
$$

Um gráfico de $\ln \eta$ versus $\frac{1}{T}$, segundo Canciam (2008), fornece uma reta, em que o coeficiente angular é numericamente igual à razão $\left(\frac{E_{a}}{R}\right)$ e $\quad$ o coeficiente linear, à $\ln \eta_{\infty}$.
A Equação 2 indica a dependência da viscosidade de um fluido com a temperatura e a mesma pode ser utilizada com dados experimentais da viscosidade na determinação da energia de ativação de fluxo viscoso e do parâmetro $\eta_{\infty}$. $\mathrm{O}$ conhecimento desses parâmetros permite verificar os efeitos da temperatura sobre a viscosidade, além de modelar matematicamente o comportamento reológico desse fluido (GRATÃO et al., 2004).

Com relação à energia de ativação de fluxo viscoso $\left(E_{a}\right)$, essa grandeza indica a sensibilidade da viscosidade devido à mudança de temperatura, de maneira que, quanto maior for a energia de ativação de fluxo viscoso, maior será a influência da temperatura. Ou seja, altos valores da energia de ativação de fluxo viscoso indicam uma mudança mais rápida na viscosidade com a temperatura (PEREIRA et al., 2003; SILVA, 2008).

O objetivo deste trabalho foi predizer a energia de ativação de fluxo viscoso dos líquidos iônicos imidazólicos hexafluorofosfato de 1-metil-3hexilimidazólio $\quad\left([\mathrm{HMIM}]\left[\mathrm{PF}_{6}\right]\right) \quad$ e bis(trifluorosulfonilo)imideto de 1-butil-3metilimidazólio ([BMIM][Tf $\left.\left.f_{2} \mathrm{~N}\right]\right)$. A predição da energia de ativação de fluxo viscoso utilizou a análise de regressão linear de dados da viscosidade de cada um dos líquidos 
iônicos imidazólicos em função da temperatura à pressão de 0,1MPa. Esses dados foram encontrados em outro trabalho.

\section{MATERIAIS E MÉTODOS}

A Tabela 1 indica os dados da viscosidade dos líquidos iônicos imidazólicos [HMIM] $\left[\mathrm{PF}_{6}\right]$ e $[\mathrm{BMIM}]\left[\mathrm{Tf}_{2} \mathrm{~N}\right]$, à pressão de 0,1 MPa, em função da temperatura. Esses dados foram adaptados do trabalho de Harris e coautores (2007).

Para a análise de regressão linear foram consideradas as Equações 3, 4, 5, 6, 7, $8,9,10,11$ e 12. Essas equações são adaptações do trabalho de Triola (2008).

A Equação 3 e a Equação 8 determinam, respectivamente, a razão $\frac{E_{a}}{R} \mathrm{e}$ $\ln \eta_{\infty}$

Tabela 2. Efeito da temperatura na viscosidade dos líquidos iônicos imidazólicos $[\mathrm{HMIM}]\left[\mathrm{PF}_{6}\right] \mathrm{e}$

\begin{tabular}{|c|c|c|}
\hline Temperatura (K) & $\begin{array}{c}\text { Viscosidade do } \\
\text { [HMIM] }\left[\mathrm{PF}_{6}\right] \\
(\mathrm{Pa} . \mathrm{s})\end{array}$ & $\begin{array}{c}\text { Viscosidade do } \\
{[\mathrm{BMIM}]\left[\mathrm{Tf}_{2} \mathrm{~N}\right]} \\
(\mathrm{Pa} . \mathrm{s})\end{array}$ \\
\hline 273,15 & 3,924 & 0,192 \\
\hline 278,15 & 2,428 & 0,140 \\
\hline 283,15 & 1,566 & 0,106 \\
\hline 288,15 & 1,036 & 0,082 \\
\hline 293,15 & 0,705 & 0,064 \\
\hline 298,15 & 0,497 & 0,051 \\
\hline 303,15 & 0,357 & 0,042 \\
\hline 313,15 & 0,197 & 0,029 \\
\hline 323,15 & 0,118 & 0,021 \\
\hline 333,15 & 0,075 & 0,016 \\
\hline 343,15 & 0,050 & 0,012 \\
\hline 348,15 & 0,041 & 0,011 \\
\hline 353,15 & 0,035 & 0,010 \\
\hline
\end{tabular}

Fonte: Adaptado de Harris et al. (2007).

$$
\frac{E_{a}}{R}=\frac{(n \cdot c)-d}{(n \cdot f)-g}
$$

Em que:

$$
\begin{aligned}
& c=\sum\left(\frac{1}{T} \cdot \ln \eta\right) \\
& d=\left(\sum \frac{1}{T}\right) \cdot \sum \ln \eta
\end{aligned}
$$

$$
\begin{aligned}
& f=\sum\left(\frac{1}{T}\right)^{2} \\
& g=\left(\sum \frac{1}{T}\right)^{2}
\end{aligned}
$$

$$
\ln \eta_{\infty}=\frac{(n \cdot f \cdot h)-(d \cdot i)}{(n \cdot f)-g}
$$

Em que:

$$
h=\sum \ln \eta
$$

$$
i=\sum \frac{1}{T}
$$

A Equação 11, também adaptada de Triola (2008), fornece o coeficiente de correlação $\left(r^{2}\right)$ para a análise de regressão linear dos dados de $\ln \eta$ versus $\frac{1}{T}$.

$$
r^{2}=\frac{(n \cdot c)-d}{\sqrt{[(n \cdot f)-g] \cdot\left[(n \cdot j)-h^{2}\right]}}
$$




$$
j=\sum(\ln \eta)^{2}
$$

Sendo $n$ correspondente ao número de dados amostrais emparelhados.

De acordo com a Tabela 1, n corresponde a 13.

A multiplicação da razão $\frac{E_{a}}{R}$ por $R$ fornece como resultado a energia de ativação de fluxo viscoso $\left(E_{a}\right)$. Para esse cálculo, foi considerado que a constante dos gases ideais $(R)$ é igual a $8,314 \times 10^{-3} \mathrm{~kJ} \cdot \mathrm{mol}^{-1} \cdot \mathrm{K}^{-1}$.

\section{RESULTADOS E DISCUSSÃO}

A Tabela 2 indica os resultados obtidos através das Equações 3, 8 e 11. Enquanto que a Tabela 3 fornece os valores obtidos para $\eta_{\infty}$ (em Pa.s) e $E_{a}\left(\mathrm{em} \mathrm{kJ} \cdot \mathrm{mol}^{-1}\right)$.

A Figura 1 ilustra o gráfico de $\ln \eta$ versus $\frac{1}{T}$ para os líquidos iônicos imidazólicos estudados.

Tabela 2. Resultados da análise de regressão linear

\begin{tabular}{cccc}
\hline Substância & $\ln \eta_{\infty}$ & $\frac{E_{a}}{R}(\mathrm{~K})$ & $r^{2}$ \\
& $($ Pa.s $)$ & & \\
\hline$[\mathrm{HMIM}]\left[\mathrm{PF}_{6}\right]$ & $-19,3835$ & 5607,5384 & 0,9971 \\
{$[\mathrm{BMIM}]\left[\mathrm{Tf}_{2} \mathrm{~N}\right]$} & $-14,6528$ & 3509,0692 & 0,9966 \\
\hline
\end{tabular}

Fonte: Autor.
Tabela 3. Valores obtidos para $\eta_{\infty}$ e $E_{a}$

\begin{tabular}{ccc}
\hline Substância & $\eta_{\infty}($ Pa.s $)$ & $E_{a}\left(\mathrm{~kJ} \cdot \mathrm{mol}^{-1}\right)$ \\
\hline$[\mathrm{HMIM}]\left[\mathrm{PF}_{6}\right]$ & $3,8182 \times 10^{-9}$ & 46,6211 \\
{$[\mathrm{BMIM}]\left[\mathrm{Tf}_{2} \mathrm{~N}\right]$} & $4,3288 \times 10^{-7}$ & 29,1744 \\
\hline
\end{tabular}

Fonte: Autor.

Figura 1: Gráfico de $\ln \eta$ versus $\frac{1}{T}$ para os líquidos iônicos imidazólicos estudados

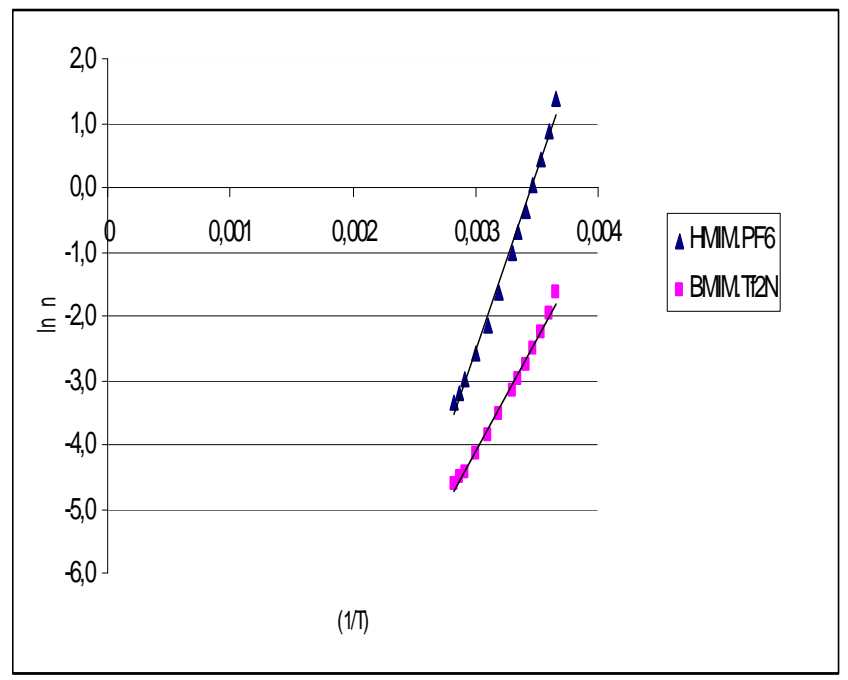

Fonte: Autor.

Foi realizada uma pesquisa na literatura e constatou-se a ausência de valores experimentais para a energia de ativação de fluxo viscoso dos líquidos iônicos imidazólicos $[\mathrm{HMIM}]\left[\mathrm{PF}_{6}\right]$ e $[\mathrm{BMIM}]\left[\mathrm{Tf}_{2} \mathrm{~N}\right]$.

Dessa maneira, dificulta a comparação dos resultados obtidos neste trabalho.

A Tabela 4 relaciona os valores da energia de ativação de fluxo viscoso de alguns materiais líquidos, encontrados na literatura. 
Tabela 4. Energia de ativação de fluxo viscoso de alguns materiais líquidos

\begin{tabular}{cccc}
\hline Materiais & $E_{a}\left(\mathrm{~kJ}^{\mathrm{mol}}{ }^{-1}\right)$ & $\begin{array}{c}\text { Faixa de } \\
\text { temperatura } \\
\left({ }^{\circ} \mathrm{C}\right)\end{array}$ & Referência \\
\hline $\begin{array}{c}\text { Azeite de } \\
\text { oliva }\end{array}$ & 27,7778 & 20 a 70 & Canciam \\
Óleo bruto & 51,1468 & -15 a 80 & Canciam \\
de & & & $(2011)$ \\
mamona \\
cultivar \\
BRS-149 \\
Nordestina
\end{tabular}

Comparando as Tabelas 3 e 4, pode-se observar que o valor da energia de ativação de fluxo viscoso do líquido iônico $[\mathrm{BMIM}]\left[\mathrm{Tf}_{2} \mathrm{~N}\right]$ é próximo ao valor da energia de ativação de fluxo viscoso do azeite de oliva. Enquanto que o valor da energia de ativação de fluxo viscoso do líquido iônico [HMIM] $\left[\mathrm{PF}_{6}\right]$ é próximo ao valor da energia de ativação de fluxo viscoso do óleo bruto de mamona cultivar BRS-149 Nordestina.

Segundo Ribeiro e coautores (2005), pela Teoria do Estado Ativado de Eyring, quanto maior for a viscosidade, maior será a barreira potencial (energia de ativação de fluxo viscoso) que uma molécula terá que vencer a fim de "saltar" para uma vacância adjacente.

Por sua vez, maiores valores da energia de ativação de fluxo viscoso implicam que a viscosidade é relativamente mais sensível à mudança de temperatura (PEREIRA et al., 2003).
Dessa forma, os resultados sugerem que o valor da energia de ativação de fluxo viscoso do líquido iônico $[\mathrm{HMIM}]\left[\mathrm{PF}_{6}\right]$ é considerado alto em relação ao valor da energia de ativação de fluxo viscoso do líquido iônico $[\mathrm{BMIM}]\left[\mathrm{Tf}_{2} \mathrm{~N}\right]$, implicando que a viscosidade é relativamente mais sensível à mudança de temperatura. Ou seja, pequenas variações de temperatura são necessárias para modificar rapidamente a viscosidade do líquido iônico [HMIM] $\left[\mathrm{PF}_{6}\right]$ em comparação com o líquido iônico [BMIM][Tf $2 \mathrm{~N}]$.

Considerando a temperatura, a própria equação do tipo Arrhenius (Equação 1) indica a dependência da viscosidade, de maneira que o aumento da temperatura provoca uma diminuição da viscosidade. Essa redução de viscosidade com o aumento da temperatura é atribuído, segundo Granjeiro e coautores (2007), ao aumento das distâncias intermoleculares provocadas pelo aquecimento. $\mathrm{O}$ aumento das distâncias reduz as forças atrativas intermoleculares, diminuindo a viscosidade.

De acordo com Triola (2008), o coeficiente de correlação $\left(r^{2}\right)$ mede o grau de relacionamento linear entre os valores emparelhados das variáveis dependente e independente em uma amostra, avaliando assim, a qualidade do ajuste. $\mathrm{O}$ mesmo autor comenta que coeficientes de correlação 
próximos da unidade indicam que é melhor a qualidade do ajuste da função aos pontos do diagrama de dispersão.

Lira (2004) comenta que para valores do coeficiente de correlação maiores ou iguais a 0,90 e menores que 1,0 , a correlação linear é classificada como muito forte.

Dessa maneira, para os líquidos iônicos imidazólicos estudados, a correlação linear é classificada como muito forte.

\section{CONCLUSÃO}

A modelagem de dados experimentais da viscosidade em função da temperatura permitiu predizer os valores da energia de ativação de fluxo viscoso dos líquidos iônicos [HMIM] $\left[\mathrm{PF}_{6}\right]$ (hexafluorofosfato de 1-metil3-hexilimidazólio) e $[\mathrm{BMIM}]\left[\mathrm{Tf}_{2} \mathrm{~N}\right]$ (bis(trifluorosulfonilo)imideto de 1-butil-3metilimidazólio).

$\mathrm{O}$ valor encontrado para a energia de ativação de fluxo viscoso do $[\mathrm{HMIM}]\left[\mathrm{PF}_{6}\right]$ foi de 46,6211 kJ.mol ${ }^{-1}$, enquanto que para o [BMIM] $\left[\mathrm{Tf}_{2} \mathrm{~N}\right]$, o valor foi de 29,1744 $\mathrm{kJ} \mathrm{mol}^{-1}$. Esses resultados sugerem que o [HMIM] $\left[\mathrm{PF}_{6}\right]$ apresenta uma maior sensibilidade da viscosidade quanto ao aumento de temperatura, quando comparado ao $[\mathrm{BMIM}]\left[\mathrm{Tf}_{2} \mathrm{~N}\right]$.

A ausência na literatura de valores experimentais para a energia de ativação de fluxo viscoso dos líquidos iônicos imidazólicos estudados dificultou a comparação com os valores preditos.

Comparando com outros trabalhos, pode-se observar que o valor da energia de ativação de fluxo viscoso do líquido iônico $[\mathrm{BMIM}]\left[\mathrm{Tf}_{2} \mathrm{~N}\right]$ encontra-se próximo ao valor da energia de ativação de fluxo viscoso do azeite de oliva. Enquanto que o valor da energia de ativação de fluxo viscoso do líquido iônico $[\mathrm{HMIM}]\left[\mathrm{PF}_{6}\right]$ é próximo ao valor da energia de ativação de fluxo viscoso do óleo bruto de mamona cultivar BRS-149 Nordestina.

Nas análises de regressão linear dos líquidos iônicos imidazólicos estudados, os valores do coeficiente de correlação encontraram-se próximos da unidade, caracterizando como correlações lineares muito fortes.

\section{REFERÊNCIAS}

ANDO, R. A.; SANTOS, P. S. Interação de $\mathrm{SO}_{2}$ e $\mathrm{CS}_{2}$ com líquidos iônicos imidazólicos. In: Reunião Anual da Sociedade Brasileira de Química, 34. 2011, Florianópolis. Anais... Florianópolis: SBQ, 2011.

CANCIAM, C. A. Efeito da temperatura na viscosidade de soluções de sacarose. In: SANTOS JÚNIOR, G.; ALMEIDA, D. M.; MICHALOSKI, A. O. (ed.). Série em Ciência e Tecnologia de Alimentos: agroindústria, energia e meio ambiente. Ponta Grossa: UTFPR, 2008. 
CANCIAM, C. A. Efeito da temperature na viscosidade de óleos vegetais refinados. Publicatio UEPG - Ciências Exatas e da Terra, Ciências Agrárias e Engenharias, v. 16, n. 1, p. 7-12, 2010.

CANCIAM, C. A. Predição do coeficiente de expansão térmica e da energia de ativação do óleo bruto de mamona através da aplicação da análise de regressão linear. E-xacta, v. 4, n. 3, p. 7-18, 2011.

CONSORTI, C. S.; SOUZA, R. F.;

DUPONT, J. Líquidos iônicos contendo o cátion dialquilimidazólio: estrutura, propriedades físico-químicas e comportamento em solução. Revista Química Nova, v. 24, n. 6, p. 830-837, 2001.

DUPONT, J. On the solid, liquid and solution structural organization of imidazolium ionic liquids. Journal of the Brazilian Chemical Society, v. 15, n. 3, p. 341-350, 2004.

GIAP, S. G. E. The hidden property of Arrhenius-type relationship: viscosity as a function of temperature. Journal of Physical Science, v. 21, n. 1, p. 29-39, 2010.

GRANJEIRO, A. A.; QUEIROZ, A. J. M.; FIGUEIREEDO, R. M. F.; MATA, M. E. R.

M. C. Viscosidades de polpas concentradas de figo-da-Índia. Revista Brasileira de

Agrociência, v. 13, n. 2, p. 219-224, 2007.

GRATÃO, A. C. A. BERTO, M. I.;

SILVEIRA JÚNIOR, V. Reologia do açúcar líquido invertido: influência da temperatura na viscosidade. Revista Ciência e Tecnologia de Alimentos, v. 24, n. 4, p. 652-656, 2004.

HARRIS, K. R.; KANABUKO, M. WOOLF, L. A. Temperature and pressure dependence of the viscosity of the ionic liquids 1-hexyl-3methylimidazolium hexafluorophosphate and 1-butyl-3-methylimidazolium bis(trifluoromethylsulfonyl)imide. Journal of
Chemical \&Engineering Data, v. 52, n. 3, p. 1080-1085, 2007.

LIMA, P. G. Micro-ondas na síntese de líquidos iônicos. Revista Virtual de Química, v. 3, n. 4, p. 325-338, 2011.

LIRA, S. A. Análise de correlação: abordagem teórica e de construção dos coeficientes com aplicação. Dissertação de Mestrado em Ciências da Universidade Federal do Paraná, 2004. 196p.

MIGLIORINI, M. V. Líquidos iônicos para a preparação de híbridos de sílica e suas aplicações na formação de compósitos poliméricos. Dissertação de Mestrado em Química da Universidade Federal do Rio Grande do Sul, 2009. 90p.

OLIVEIRA, R. C.; BARROS, S. T. D.; ROSSI, R. M. Aplicação da metodologia Bayesiana para o estudo reológico da polpa de uva. Revista Brasileira de Produtos Agroindustriais, v. 11, n. 1, p. 73-80, 2009.

PEREIRA, E. A.; QUEIROZ, A. J. M.; FIGUEIRÊDO, R. M. F. Comportamento reológico de mel da abelha uruçu (Melipona scutellaris, L.). Revista de Ciências Exatas e Naturais, v. 5, n. 2, p. 179-186, 2003.

RIBEIRO, L. D.; CRUZ, S. R. A.; REIS, R. A. Cálculo de viscosidade de misturas não eletrolíticas. In: Congresso Brasileiro de Engenharia Química em Iniciação Científica, 6. 2005, Campinas. Anais... Campinas: Unicamp, 2005.

RODRIGUES, F. Espectroscopia Raman de líquidos iônicos imidazólicos: interações interiônicas, organização estrutural e efeitos de micro-ambiente. Tese de Doutorado em Química da Universidade de São Paulo, 2010. 173p.

ROGERS, R. D.; SEDDON, K. R.; VOLKOV, S. Green Industrial Applications 
of Ionic Liquids. Kluwer Academic

Publishers, Dordrecht, 2002. 553p.

SHAMES, I. H. Mecânica dos Fluidos volume 1. Editora Edgard Blücher, São Paulo, 1999. 192p.

SILVA, A. A.; PAREDES, M. L. L.; REIS, R. A. Modelagem de viscosidade de misturas não eletrolíticas. In: Congresso Brasileiro de Engenharia Química em Iniciação Científica, 7. 2007, São Carlos. Anais... São Carlos: UFSCAR, 2007.

SILVA, F. M.; LACERDA, P. S. B.; JONES JUNIOR, J. Desenvolvimento sustentável e Química Verde. Química Nova, v. 28, n. 1, p. 103-110, 2005.

SILVA, S. A. Estudo do comportamento reológico dos adesivos hot melt PSA e a sua relação com a composição e as propriedades adesivas. Dissertação de Mestrado em Engenharia Química da Universidade Federal do Rio Grande do Sul, 2008. 123p.

TRIOLA, M. F. Introdução a Estatística.

Livros Técnicos e Científicos, Rio de Janeiro, 2008. 410p.

VALENTE, M. V. Polimerização de PMMA em microemulsão com Lis tensiactivos. Dissertação de Mestrado em Engenharia Química da Universidade de Aveiro, 2008. 96p. 